\title{
"Analysis of binding properties of integrase protein to find out potential drug candidate for HIV-2 through insilico molecular docking"
}

\author{
Vijay Laxmi Saxena ${ }^{1, *}$ Shrasti Gupta ${ }^{1}$, Shweta Maurya ${ }^{2, *}$ \\ Bioinformatics Infrastructure Facility Centre of D.B.T, Dept of Zoology, D.G (P.G.), College, Kanpur \\ Bioinformatics Infrastructure Facility Centre of D.B.T, D.G (P.G.), College, Kanpur \\ CSJM, Kanpur university, Kalyanpur, Kanpur
}

\begin{abstract}
Human immuno deficiency virus is a lentivirus (slowly replicating virus) that cause acquired immuno deficiency syndrome "(AIDS ). HIV attached to the human CD4 receptor and leads to the infection. The most notable, the T-helper cells which plays a central role in immune response are disabled and killed during the typical course of infection.HIV-2 infection is characterised by higher CD4 cell count \& lower viral RNA level. The main objective of this project work is to find out potential compound that can help to interrupt the function of $\boldsymbol{H I V - 2 i n t e g r a s e ~ ( I N ) ~ p r o t e i n . ~ I n ~ t h i s ~ s t u d y , ~ w e ~ e m p l o y e d ~ t h e ~ i n t e g r a s e ~ p r o t e i n ~ s e q u e n c e ~}$ to build suitable drug by homology modeling, which has been checked for high reliability by verify score ,energy minimization for stability and active side prediction. Its accuracy has been verified by the Saves server and Ramachandran plot. The model structure was employed for docking. Known inhibitor taken from the literature docked, and docked at the binding site. Obtain molecule 2'-Deoxyuridylic acid,2-(Ethoxymethyl)-4(4-Fluorophenyl)-3-[2-(2-Hydroxyphenoxy)Pyrimidin-4-yl]isoxazol-5(2H)-one, $\{4-[2,2-B i S(5$ methyl-1,2,4oxadiazole-3-YL)-3-phenyl pyro]phenyl\}sulfamic acid,2-(2-\{2-[(Biphenyl-4-ylmethyl)-amino]-3-Meracaptopentanoylamino]-acetylamino)-3-methyl-butaric acid methyl ester, $N$-[2-(2,4-diaminopyrido[2,3-d]pyrimidin7-yl)-2-methylpropyl]-4-phenoxybenzamide have been given score of $-136.401 \mathrm{kcal} / \mathrm{mol},-143.584 \mathrm{kcal} / \mathrm{mol}$, $136.863 \mathrm{kcal} / \mathrm{mol},-135.008 \mathrm{kcal} / \mathrm{mol},-156.243 \mathrm{kcal} / \mathrm{mol}$.

Keywords: HIV-2,CD4 receptor,Integraseprotein,Computer aided drug designing, Docking.
\end{abstract}

\section{Introduction:-}

The human immunodeficiency virus is the causal agent of AIDS. AIDS morbidity and mortality have led to efforts to identify effective inhibitors of the replication of this virus.Integration of retroviruses like the Human Immunodeficiency Virus-1 (HIV-1) \& Human Immunodeficency virus-II (HIV-II) establishes a provirus in the host genome, embodying the point-of-no-return in the viral replication cycle.[13]. Viral replication is driven by a molecular motor consisting of the three viral enzymes: the reverse transcriptase, protease and integrase (IN).(2).Our study is mainly deals with Integrase enzyme.Integrase possesses two major catalytic activities: an endonucleolytic cleavage at each 3'-OH extremities of the viral genome, named 3'processing, and a strand transfer reaction leading to the insertion of the processed viral DNA into the target DNA by a trans-esterification mechanism. These catalytic functions of the integrase are essential for the overall integration process and have thus been the object of intensive pharmacological research.[13]

Drugdesigning is a time consuming and expensive process. The first stages of this process are lead discovery and lead optimisation.Traditionally lead compound have been discovered serendipitously, by chemically modifying andimproving existing drugs orby isolating the active ingredients in herbal remedies. More recently, pharmaceutical companies have focussed on high throughput screening (HTS). This involvesscreening a large chemical libraryagainst a protein target.[1]The choice of a drug target is primarily made on a biological and biochemical basis. The ideal target macromolecule for structure-based drug design is one that is closely linked to human disease and binds a small molecule in order to carry out a function. The target molecule usually has a well defined binding pocket. In our study the drug target is integarse enzyme.

\section{TYPES OF HIV AND DIFFERENCES BETWEEN THEM:-}

Two types of HIV have been characterized: HIV-1 and HIV-2. HIV-1 is the virus that was initially discovered and termed both LAV and HTLV-III. It is more virulent, more infectiveand is the cause of the majority of HIV infections globally. The lower infectivity of HIV-2 compared to HIV-1 implies that fewer of those exposed to HIV-2 will be infected per exposure. Because of its relatively poor capacity for transmission, HIV-2 is largely confined to West AfricaSimilar in many ways, there are important differences between HIV-1 and HIV-2 that provide insights into virus evolution, tropism and pathogenesis. Major differences include reduced pathogenicity ofHIV-2 relative to HIV-1, enhanced immune control of HIV-2 infection and often some 
degree of CD4-independence.Despite considerable sequence and phenotypic differences between HIV-1 and 2 envelopes, structurally they are quite similar. Both membrane-anchored proteins eventually form the 6-helix bundles from the $\mathrm{N}$-terminal and $\mathrm{C}$-terminal regions of the ectodomain, which is common to many viral and cellular fusion proteins and which seems to drive fusion. HIV-1IIIB gp41 helical regions can form more stable 6-helix bundles than HIV-2SBL gp41 helical regions ; however HIV-2 fusion occurs at a lower threshold temperature $\left(25^{\circ} \mathrm{C}\right)$. [11] HIV type I (HIV-1) and type $2(\mathrm{HIV}-2)$ are very closely related but differ in pathogenicity, natural history and therapy. HIV-1 is more easily transmitted and consequently accounts for the vast majority of global HIV infections. The less transmissible HIV-2 was thought to be largely confined to West Africa (where it is thought to have originated)but has spread to parts of Europe and India. Whencompared to HIV-1, HIV-2 infected individuals have a much longer asymptomatic stage, slower progression to AIDS, slower decline in CD4 countlower mortality, lower rate of vertical transmissionand smaller gains in CD4 count in response to antiretroviral treatment (ART).Serologic reactivity to HIV-1 and HIV-2 (HIV-1/2) has also increased in HIV-2 endemic areas over the past decade. In terms of antiretroviral drug regimens, HIV -2 is intrinsically resistant to non nuclesoside reverse transcriptase inhibitors (NNRTI) such as nevirapine and efavirenz and not all the protease inhibitors (PIs) provide good viral suppression15. The genome of HIV-2 is 9,671 nucleotides is long,whereas HIV-1 is isolated about 9200 nucleotides long. The genetic organization of HIV-2 is analogous to HIV-1.[12]

\section{5'Ltr-gag-pol-central region-env-orf-F-3'Ltr}
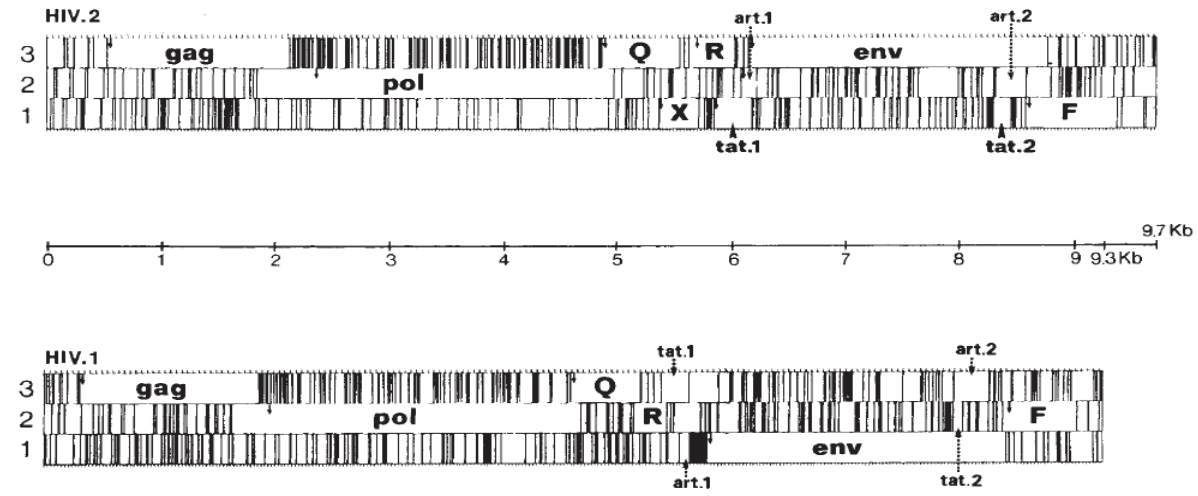

FIG :1HIV-2 has been found to be more infectious in later stages,causing a number of alignments in a very short spans.[11]

\section{Comparision of HIV-1 and HIV-2 characteristics}

\begin{tabular}{|l|l|l|}
\hline \multicolumn{1}{|c|}{ Characteristics } & \multicolumn{1}{c|}{ HIV-1 } & \multicolumn{1}{c|}{ HIV-2 } \\
\hline Infectivity & High & Low \\
\hline Virulence & High & Low \\
\hline Heterosexual spread & Higher & Lower \\
\hline Vertical Transmission & $20-50 \%$ & $\leq 5 \%$ \\
\hline Genetic Diversity & - & Lower \\
\hline Prevalence & Global & West Africa \\
\hline Time to AIDS & $\leq 10$ Years & $\geq 20$ Years \\
\hline
\end{tabular}

\section{Materials And Methodology :-}

\section{PROTEIN MODEL:-}

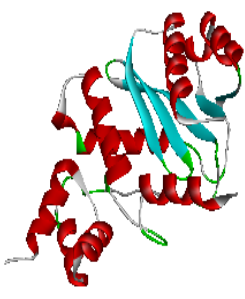

(A)

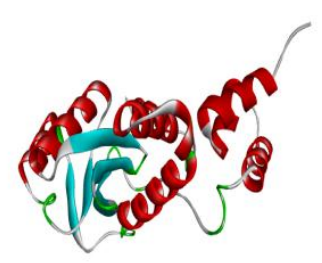

(B)

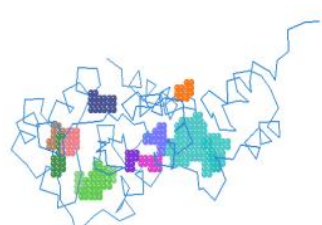

C)

FIG1:(A)3D structure of integrase protein sequence, Red colours shows helical ,blue colurs shows sheet and green colours shows coiling in the structure of protein. FIG:(B)The model of integrase protein have been shown below which have minimum energy and is most stable. FIG:(C) 9active sites for the ligand binding sites. Among of them two active sites have larger pocket size .His, Tyr, Ser, Asn, Val, Gyl, Lys, Cys, Glu, Ala, Metamino acid residues are present at largest pocket site 


\begin{tabular}{|l|c|l|l|c|}
\hline Sites & Min cords & Max coords & Site volume & Protein volume \\
\hline Site 1(Blue) & $(19,-2,-6)$ & $(1,-6,-17)$ & $3017\left[\AA^{3}\right]$ & $19894\left[\AA^{3}\right]$ \\
\hline Site 2(Green) & $(1,-6,-17)$ & $(15,7,-4)$ & $118\left[\AA^{3}\right]$ & $19894\left[\AA^{3}\right]$ \\
\hline
\end{tabular}

BEST INTRACTION SHOWS WITH LIGAND 30:- N-[2-(2,4-diaminopyrido[2,3-d]pyrimidin-7-yl)-2methylpropyl]-4-phenoxybenzamide

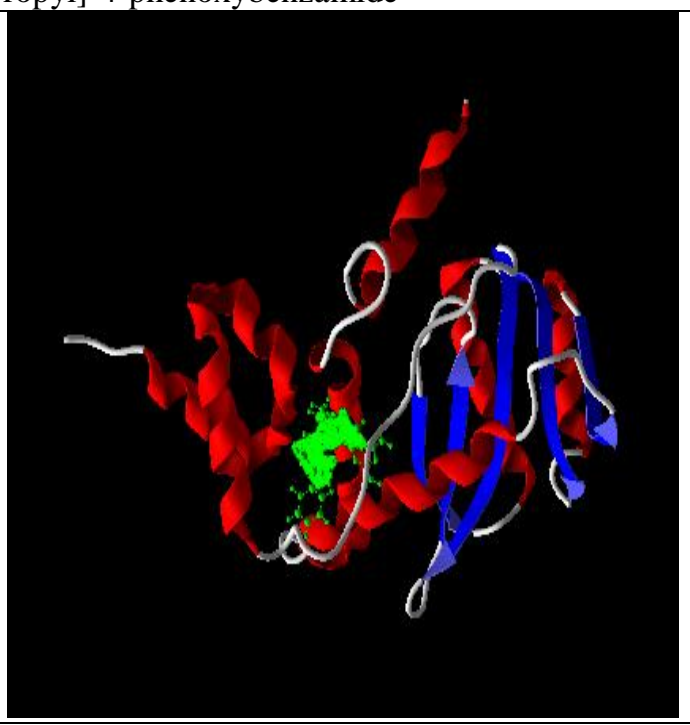

(a)

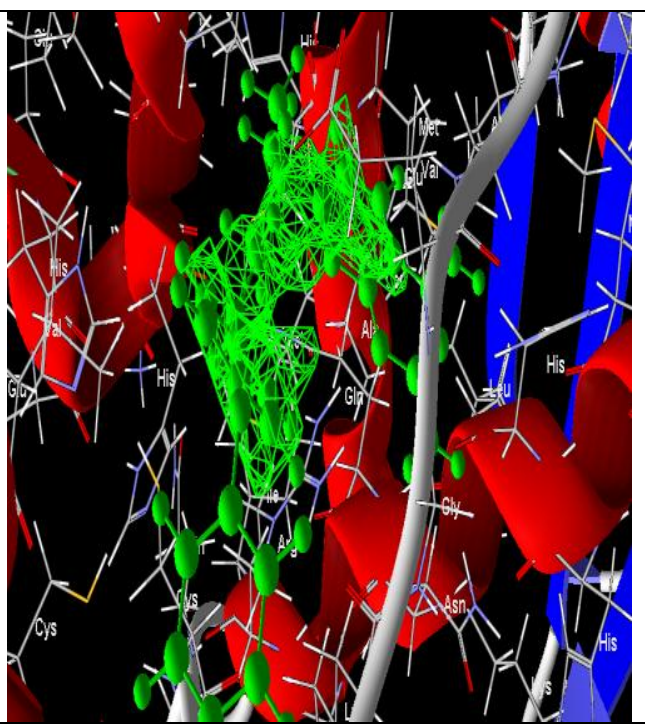

(b)

FiG2:-(a)LigandN-[2-(2,4-diaminopyrido[2,3-d]pyrimidin-7-yl)-2-methylpropyl]-4-

phenoxybenzamidebinding with integrase protein. (b) Structure containing His, Met, Glu, Arg, Val amino acid residues at the binding site of ligand.

\begin{tabular}{|l|l|l|l|}
\hline Cavity of active site & Surface of protein & Moldock Score & H-Bond \\
\hline $41.472(\mathrm{Vol})$ & 160 & -156.243 & -7.07561 \\
\hline
\end{tabular}

\section{Result And Discussion:-}

Human Immuno deficiency virus cause AIDS which leads to life threatening opportunistic infections..Integrase protein sequence has been retrived from NCBI and the structure is modeled by Raptor X server. Raptor X server generated models of Integrase protein, model has been considered best because it has maximum core region, and minimum disallowed region and minimum energy. Then we using Saves server to verify the structure by verify score plot, procheck and Ramachandran plot. After this active site have been predicted using the $\mathrm{P}$ - site finder which resulted in to 9 active site each having specific volume, area and amino acid residues. the site 1 which having maximum volume has following amino acid His(158), tyr(16), ser(18), asn (161), val (38) ,cys (44), lys (47), gly (48), glu (49), ala (50), gln (162), arg (188), cys (183), met (184), lys (187). Active site is the site were the ligand binds disturbed the activity of receptor. To analyse the active site of protein .It is selected their largest pocket size withe higher number of site volume and higher number of residues present at the active site. To the prediction of protein ligand interaction, first chemical compounds were selected from drug bank and sdf (structure data file) format from Zinc database and validated to the Lipinski rule of five. It's a rule for analysing the pysio-chemical property of drug and analysed that a drug is more likely to membrane permeable and easily absorbed by the body. All selected compound follow Lipinski rules of five.Towards finding suitable inhibitors for Integrase protein the binding energy of prescribe drug for HIV 2 has been done by Molegro Virtual Docker is an integrated platform for predicting protein - ligand interaction. It is analysed docking from MVD the Mol dock score value and hydrogen bonds value shows higher binding affinity with the lower binding energy. N-[2-(2,4-diaminopyrido[2,3-d]pyrimidin-7-yl)-2-methylpropyl]-4phenoxybenzamide have energy binding value $\mathbf{( - 1 5 6 . 2 3 4 3} \mathbf{~ k c a l} / \mathbf{m o l})$ and hydrogen bindingvalue (7.07561). The bioactivity of $\mathrm{N}$-[2-(2,4-diaminopyrido[2,3-d]pyrimidin7-yl)-2-methylpropyl]-4phenoxybenzamide shows maximum positive value of dug likeness property it shows positive number of kinase inhibitor, Enzyme inhibitor and higer score of GPCR ligand score.

\section{Conclusion:-}

In study of HIV-2 integrase protein with various ligand determine and the interaction between Inegrase protein and selected ligand that bind on active site of the Integrase enzyme ,although docking process is very complicated because its depends on various parameters the main resultant obtained by Molegro virtual docker for identify the suitable HIV-2 integrase inhibitor in all the 30 ligands which are docked with the integrase only 
5 numbers of ligand given the minimum energy and these are2'-Deoxyuridylic acid,2-(Ethoxymethyl)-4-(4 Fluorophenyl)-3-[2-(2-Hydroxyphenoxy)Pyrimidin-4-yl]isoxazol-5(2H)-one, $\{4-[2,2-\mathrm{BiS}(5$ methyl1,2,4-oxadiazole-3-YL)-3-phenyl pyro]phenyl $\}$ sulfamic acid,2-(2-\{2-[(Biphenyl-4-ylmethyl)-amino]-3Meracapto-pentanoylamino -acetylamino)-3-methyl-butaric acid methyl ester,N-[2-(2,4-diaminopyrido[2,3d]pyrimidin-7-yl)-2-methylpropyl] phenoxybenzamide have been given score of $-136.401 \mathrm{kcal} / \mathrm{mol},-143.584$ $\mathrm{kcal} / \mathrm{mol},-136.863 \mathrm{kcal} / \mathrm{mol},-135.008 \mathrm{kcal} / \mathrm{mol},-156.243 \mathrm{kcal} / \mathrm{mol}$. Out of these 5 ligands, it is analyased that N-[2-(2,4-diaminopyrido[2,3- d]pyrimidin-7-yl)-2- methylpropyl]-4- phenoxybenzamideis potential Integrase inhibitor. Perhaps the ultimate solution is to develop a potential drug candidate against this devastating epidemic.

\section{References:-}

[1] Alasdair T.R. Laurie and Richard M JacksonMethods for prediction of protein ligand binding sites for structure based drug designing and virtual screenings.Bentham science publishers Ltd (2006)

[2] Alasdair T.R. Laurie and Richard M Jackson Q-SiteFinder: an energy-based method for the prediction of protein-ligand binding sites.

[3] ALKA DWIVEDI, VIJAY LAXMI SAXENA In silicodrug designing of protease inhibitors to find out potential drug candidate for HIV-1.Computational Biology and Bioinformatics, 1(3): 10-14 2013;

[4] Amy C. AndersonThe Process of Structure-Based Drug Design.Chemistry \& Biology, Vol. 10, 787-797, September, 2003,

[5] Batul Diwan*, Rupali Saxena and Archana Tiwari HIV-2 and its role in conglutinated approach towards Acquired Immunodeficiency Syndrome (AIDS) Vaccine Development, SpringerPlus ,Vol 2:7, 2013,

[6] Bineeta Kashyap, Hitender Gautam, Sanjim Chadha and Preena Bhalla DELAYED PROGRESSION AND INEFFICIENT TRANSMISSION OF HIV-2.Vol 41 No. 3 May 2010

[7] Fredrik BryntessonTHE LIFE CYCLE OF THE HUMAN IMMUNODEFICIENCY VIRUS.THE NEW PHILOSOPHY, JanuaryJune 2009

[8] Hans R. Gelderblom Fine Structure of HIV and SIV.

[9] Jian XuPhylogenetic Analysis of the Origin ofthe HIV virus.

[10] Koteswara Reddy Gujjula PREDICTION AND COMPARISON OF HIV-1 PROTEASE INHIBITOR BINDING ENERGIES BY VARIOUS MOLECULAR DOCKING METHODS

[11] Mirreille Guyader Michel Emerman Pierrre Sonigo Francois Clavel Luc Montagnier and Marc Alizon Genome organization and transactivation of Human immunodeficiency virus type2.Nature publishing group, Vol 326, (16 april 1987),

[12] Montaldo Chiara, Zachariah Rony*, Mansoor Homa, Varghese Bhanumati, Joanna Ladomirska, M. Manzi*, N. Wilson+, Deshpande Alaka** \& A.D. HarriesCharacteristics, immunological response \& treatment outcomes of HIV-2 compared with HIV-1 \& dual infections (HIV 1/ 2) in Mumbai. Indian J Med Res 132, December 2010, pp 683-689

[13] Olivier Delelis1, Kevin Carayon1, Ali Saïb2, Eric Deprez1 and Jean FrançoisMouscadet1 Integrase and integration: biochemical activities of HIV-1 integrase.Biomed central, 17 december,Vol 5:114, 2008,

[14] Omobolaji T. Campbell-Yesufu,1 and Rajesh T. Gandhi1,2 Update on Human Immunodeficiency Virus (HIV)-2 Infection.Clinical infectious disease, Vol 52;(6)780-787, 2011,

[15] P. LALITHA* and S. SIVAKAMASUNDARI Calculation of molecular lipophilicity and drug likeness for few heterocycles.

[16] Rita Cavaleiro1, Gregory J. Brunn2, Adriana S. Albuquerque1, Rui M. M. Victorino1, Jeffrey L. Platt3 and Ana E. Sousa1 Monocyte-mediated T cell suppression by HIV-2 envelope proteins.Eur. J. Immunol. Vol 37: 3435-3444., 2007.

[17] Stephen A Gallo1, Jacqueline D Reeves2, Himanshu Garg1, Brian Foley3,Robert W Doms2 and Robert Blumenthal 1 Kineticstudies of HIV-1 and HIV-2 envelope glycoprotein-mediated fusion.Retrovirology, Vol 3:90, 2006, 